\title{
Edge Detection in Color Images - A Comparative Study
}

\author{
Hanumanthappa M, Ph.D \\ Associate Professor \\ Dept. of Computer Science and Applications, \\ Bangalore University, Bangalore
}

\author{
S Regina Lourdhu Suganthi \\ Research Scholar \\ Dept. of Computer Science and Applications \\ Bangalore University, Bangalore
}

\begin{abstract}
The ability to detect edges from an image plays a key role in solving many problems related to computer vision. Extracting appropriate features from an image is a great challenge in the context of content based image retrieval. Some important points in an image could be used to describe the features of an object. To recognize objects from an image, it is essential that the features extracted from an image are invariant due to noise and illumination. Such points lie in high contrast regions such as edges of an object. Generally, a color image is converted to gray scale image to detect the edges of objects. In this paper two color spaces namely RGB and HSV have been considered. These color spaces individually are combined with gray plane and the effect has been discussed. It is evident from the results that by either OR-ing the $\mathrm{R}, \mathrm{G}, \mathrm{B}$ planes with the grayscale plane or by OR-ing S, V planes with the grayscale plane more edges could be detected.
\end{abstract}

\section{Index Terms}

HSV (Hue, Saturation, Value), LEBP, RGB (Red, Green, Blue), SAM

\section{INTRODUCTION}

Multimedia data, specifically image and video data acquired through digital devices installed at event venues of various governmental, educational, industrial and social organizations occupy enormous amount of storage space in the servers. Eventually these data must be available for future access based on user queries. Extracting essential features based on its content ${ }^{[6]}$ fasten the search results. Processing such queries accelerates searching and searching could be fastened, if the data acquired can be efficiently indexed. Multimedia data is high dimensional and is considerably difficult to classify due to curse of dimensionality ${ }^{[8]}$. Hence, choosing essential features from an image or video data play a significant role in indexing ${ }^{[4]}$. The feature extracted could be a point, a curve, edges, an area or any other feature ${ }^{[5]}$. This paper focuses on edge detection from color images by combining the color space with grayscale plane. Two color spaces namely RGB and HSV have been considered.

The paper is organized as follows :

Section 2 presents the literature survey of the related topics. Section 3 discusses the methodology used in the findings. Section4 presents the experimental results by comparing the combination of color planes and the grayscale plane. Section 5 concludes with scope of the work.

\section{LITERATURE SURVEY}

Data in multimedia form is ubiquitous in present times. Multimedia data possess various inherent characteristics. Mining process deals with feature extraction, indexing and ranking to facilitate various operations namely similarity search, pattern recognition and many more operations depending on the application. Indexing the data is essential ${ }^{[2]}$ to facilitate quick retrieval. Common method for indexing multimedia data is Spatial Access Methods (SAM) ${ }^{[7]}$. SAMs is a data structure based on the position and shape or spatial relationships of spatial objects. Image and series of image frames constituting video are components of multimedia data. Features that could be extracted from an image data include significant points, curves, edges, texture and shape.

Edge detection is of fundamental importance in image analysis. Operations such as identification of objects in an image, content based image segmentation require characterizing the boundaries of an object. Since edges characterize object boundaries, edge detection is vital in image processing. Edge points in an image are those pixel locations with an abrupt change in gray level. For a binary image, it is black pixels with at least one white pixel as its nearest neighbor, that is,

for pixel location $(x, y)$ such that $f(x, y)=0$ and $h(x, y)=1$, where $h(x, y)=[f(x, y) \oplus f(x \pm 1, y)]$ OR $[f(x, y) \oplus f(x, y \pm 1)]$. On the other hand, in the direction of the edge of a continuous image $f(x, y)$, its derivative assumes a local minimum. One of the edge detection method is to measure the gradient of $f(x, y)$. Gradient operators known as masks are used as edge detection operators for digital images [1]. Sobel, Prewitt, Roberts are common edge detection techniques that differ in its gradient operators.

The work by Ojala et al ${ }^{[9]}$ to describe the local structures in an image using Local Binary Patterns is proven to be simple and robust. The fusion of local edge and binary patterns known as Local Edge Binary Pattern (LEBP) ${ }^{[3]}$ is shown to be efficient in texture analysis. Here, the local edge for a neighborhood $(\mathrm{X}, \mathrm{Y})$ is defined as follows, where $\mathrm{X}$ is the number of sampling pixels on a circle and $\mathrm{Y}$ is the circle radius :

$$
\begin{array}{ll}
\mathrm{e}_{\mathrm{i}} & =\mathrm{p}_{\mathrm{i}}-\mathrm{p}_{\mathrm{c}} \\
\mathrm{e}_{\mathrm{i}+\mathrm{X} / 2} & =\mathrm{p}_{\mathrm{c}}-\mathrm{p}_{\mathrm{i}+\mathrm{X} / 2}, \mathrm{i} \in[0, \mathrm{X} / 2-1] \\
\mathrm{e}_{\mathrm{c}} & =0
\end{array}
$$

where $e_{i}$ 's are the edge values and $p_{c}$ is the gray value of the middle pixel, $\mathrm{p}_{\mathrm{i}}$ is the gray value of the sample pixel.

\section{EDGE DETECTION \\ 3.1 Edge Detection in Color Images}

Every human being perceives color in different forms and hence it is a challenge in problems related to machine vision. There are various color spaces that are used to represent the images. Generally, for detecting edges from an image, the image is converted into a grayscale image and then the gradient operators are applied. Grayscale images often do not suffice the information needed for various tasks such as identification, sorting etc.,. In the virtue of the trade-off between space and time-complexity, an 
alternate method that could be used is to consider the individual planes of the color space as a gray plane, apply the masks on these planes independently and combine the edges by OR-ing these edge pixels.

\subsubsection{RGB Image}

Let $(x, y)$ be a spatial point in an RGB image $f(x, y)$. Let $r(x, y)$ represent 1 if it is an edge point in a red plane and 0 otherwise, $\mathrm{g}(\mathrm{x}, \mathrm{y})$ represent 1 if it is an edge point in a green plane and 0 otherwise, and $b(x, y)$ represent 1 if it is an edge point in a blue plane and 0 otherwise.

Thus the edges in the combined R-plane, G-plane and B-plane could be obtained by OR-ing the three planes as specified in the following expression :

$$
\mathrm{e}(\mathrm{x}, \mathrm{y})_{\mathrm{rgb}}=\mathrm{r}(\mathrm{x}, \mathrm{y}) \text { OR g(x,y) OR b(x,y) }
$$

where $e(x, y)_{\text {rgb }}$ represents the edge point of a pixel in the RGB image $f(x, y)$. Precisely,

$\mathrm{e}(\mathrm{x}, \mathrm{y})_{\mathrm{rgb}}=\left\{\begin{array}{l}0, \text { if }(\mathrm{x}, \mathrm{y}) \text { is an edge point in neither of the three planes } \\ (\mathrm{R}, \mathrm{G}, \mathrm{B}) \\ 1, \text { if }(\mathrm{x}, \mathrm{y}) \text { is an edge point in either of the three planes }\end{array}\right.$

\subsubsection{HSV Color Space}

The color image is first converted to HSV color space. Let $(\mathrm{x}, \mathrm{y})$ be a spatial point in the HSV image $f(x, y)$. Let $s(x, y)$ represent 1 if it is an edge point in a saturation component and 0 otherwise, similarly $\mathrm{v}(\mathrm{x}, \mathrm{y})$ represent 1 if it is an edge point in a value component.

Thus for a HSV converted color image edges could be identified by OR-ing the two planes, as given by the following expression :

$$
\mathrm{e}(\mathrm{x}, \mathrm{y})_{\mathrm{hsv}}=\mathrm{s}(\mathrm{x}, \mathrm{y}) \text { OR v(x,y) }
$$

where $e(x, y)_{h s v}$ is the edge point of the pixel in an HSV color space image $f(x, y)$. Precisely,

$$
\mathrm{e}(\mathrm{x}, \mathrm{y})_{\mathrm{hsv}}=\left\{\begin{array}{l}
0, \text { if }(\mathrm{x}, \mathrm{y}) \text { is an edge point in neither of the two planes } \\
(\mathrm{S}, \mathrm{V})
\end{array}\right.
$$

\subsection{Edge Detection by combining Color planes and grayscale plane}

It is observed that more edges could be detected, if the color planes discussed in the respective color spaces are combined with the grayscale plane using the OR operation. If $\operatorname{gr}(\mathrm{x}, \mathrm{y})=1$ in a grayscale plane represents an edge point, then an edge point in the combined RGB and Grayscale plane is detected by the following operation :

$\mathrm{e}(\mathrm{x}, \mathrm{y})_{\text {all }}=\mathrm{r}(\mathrm{x}, \mathrm{y})$ OR $\mathrm{g}(\mathrm{x}, \mathrm{y})$ OR b(x,y) OR gr(x,y)

Similarly for an HSV color space and the grayscale plane, an edge point is detected using the following operation :

$\mathrm{e}(\mathrm{x}, \mathrm{y})_{\mathrm{all}}=\mathrm{s}(\mathrm{x}, \mathrm{y})$ OR v(x,y) OR $\operatorname{gr}(\mathrm{x}, \mathrm{y})$.

The subtraction of the two images with edges detected by combining color planes, the grayscale plane and the edges obtained only from color planes, that is, $\operatorname{abs}\left(\mathrm{e}(\mathrm{x}, \mathrm{y})_{\mathrm{rgb}}-\mathrm{e}(\mathrm{x}, \mathrm{y})_{\text {all }}\right)$ reveal that $\mathrm{e}(\mathrm{x}, \mathrm{y})_{\text {all }}$ identifies more edges.

\section{EXPERIMENTAL RESULTS}

Matlab Image processing toolbox has been used to perform the edge detection and image subtraction operations on the image.

\subsection{RGB Color Space}

An RGB image with multiple objects is shown in figure 1.

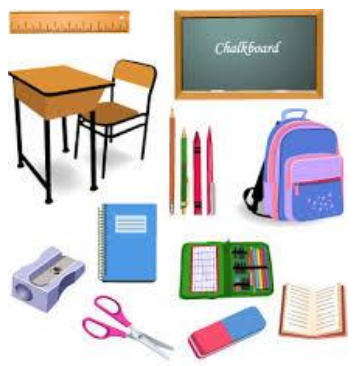

Figure 1 : An RGB Image with multiple objects

The edges detected using 'Canny' edge detection technique in the $\mathrm{R}, \mathrm{G}, \mathrm{B}$ planes are shown in figure $2 \mathrm{a}, 2 \mathrm{~b}$ and $2 \mathrm{c}$ respectively and the edges detected after converting the image into a grayscale image is shown in $2 \mathrm{~d}$.

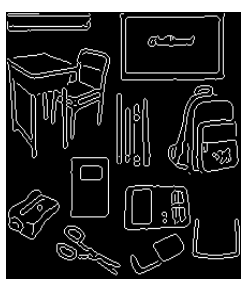

Figure 2a : Edges in R-Plane

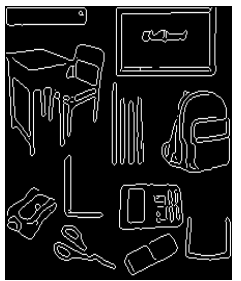

Figure 2c : Edges in B-Plane

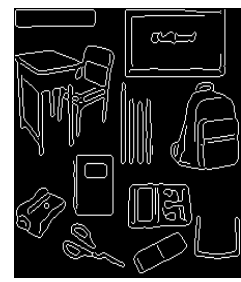

Figure 2b : Edges in G-Plane

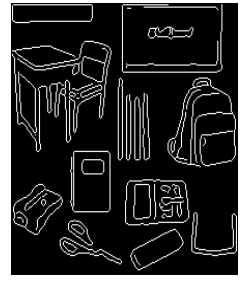

Figure 2d : Edges in Grayscale
The edges detected by combining the three color planes namely $\mathrm{R}$, $\mathrm{G}$ and $\mathrm{B}$ is shown in figure $3 \mathrm{a}$ and the edges detected by OR-ing the three planes with grayscale plane is shown in figure $3 \mathrm{~b}$.

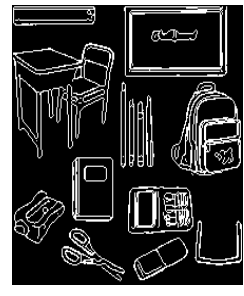

Figure 3a : Edges in R,G,B-Planes

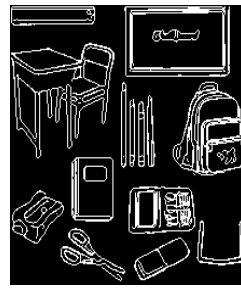

Figure 3b : Edges in RGB, Grayscale Plane
The result of image subtraction performed between the two 
images shown in figure $3 a$ and $3 b$ is presented in figure 4 . Since the resultant image comprise of non-black pixels, it is evident that more edges are present in the combination of the color and the gray planes.

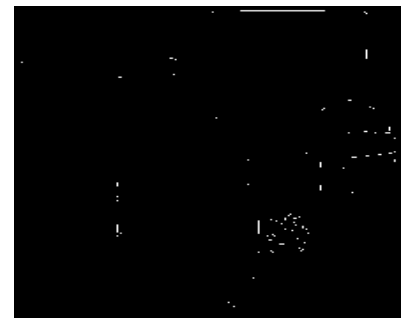

Figure 4 : Image Subtraction (Figure 3a-Figure 3b)

\subsection{HSV Color Space}

The RGB image given in figure 1 is converted to HSV color space. The edges detected using Canny edge detection technique in the Splane and the $\mathrm{V}$ - plane are shown in figure $5 \mathrm{a}$ and $5 \mathrm{~b}$.

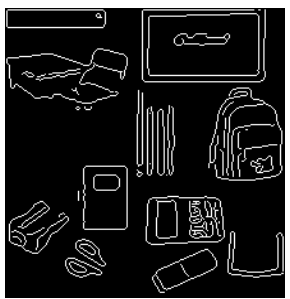

Figure 5a : Edges in S-Plane

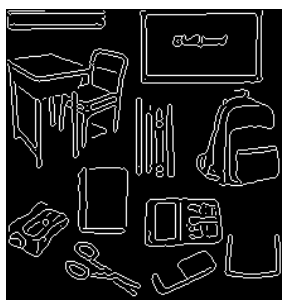

Figure 5b : Edges in V-Plane
Figure $6 \mathrm{a}$ is an image consisting of the edges detected by combining the Saturation and Value planes. Figure $6 \mathrm{~b}$ is an image with the edges detected by OR-ing the two planes with grayscale plane. The result of image subtraction given in figure 7 shows that the fusion of edges detected in the appropriate color planes and the gray plane is more than the independent operations.

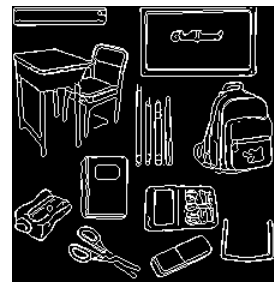

Figure 6a :

Edges in S,V Planes

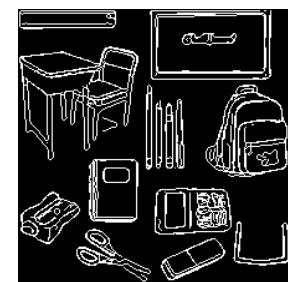

Figure 6b: Edges in S,V,Grayscale Planes

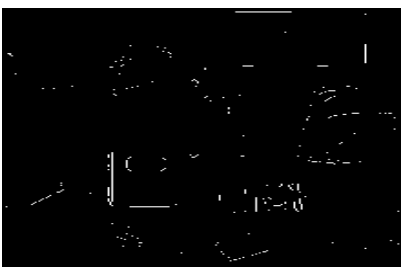

Figure 7: Image Subtraction (Figure 6a - Figure 6b)

\section{CONCLUSION}

Edge detection finds prominent role in image analysis. Edges detected from objects find its importance in image segmentation and identification of objects especially to find the shape of an object. Various edge detection techniques were developed by Researchers. The correctness and usefulness of these techniques are largely application specific. The method discussed in this paper is a simple method which could be implemented with ease. Since more edge points are identified in the fusion, it would undoubtedly be of use in many problems.

\section{REFERENCES}

[1] Anil K Jain, “Fundamentals of Digital Image Processing”, PHI Learning Private Limited, 2011, pg 347-353.

[2] D G Lowe, "Distinctive Image features from scale invariant key points", International Journal of Computer Vision, Vol. 60, no 2, pp 99-110, 2004.

[3] Junding Sun, Guoliang Fan, Xiaosheng Wu, "New Local Edge Binary Patterns For Image Retrieval ”, ICIP 2013, 4014 4018.

[4] Li-li Huang, Jin Tang,Si-bao Chen, Chris Ding and Bin Luo, "An efficient algorithm for feature selection and feature correlation", IScIDE, volume 7751 of Lecture Notes in Computer Science, page 639-646. Springer, 2012.

[5] Madhura C, Dheeraj D, "Feature Extraction for Image Retrieval using Color Spaces and GCLM", International Journal of Innovative Technology and Exploring Engineering (IJITEE), ISSN : 2278-3075, Vol. 3, Issue -2, July 2013.

[6] Ruba A. A. Salamah, "Efficient Content Based Image Retrieval", Thesis submitted to Islamic University - Gaza, Deanery of Higher Studies, 2010.

[7] Yannis Manolopoulos, Yannis Theodoridis, Vassilis J. Tsotras, "Spatial Access Methods", Advances in database systems, Vol 17, 2000, pp 117-139.

[8] Yu Jin-ping, Huang Xi-mei and Xia Xiao-yun, "Image Data Mining Technology of Multimedia", Future Computing, Communication, Control and Management Lecture Notes in Electrical Engineering Volume 144, 2012, pp 379-385.

[9] Zhou Wang, Member, Alan Conrad Bovik,, Hamid Rahim Sheikh and Eero P. Simoncelli, "Image Quality Assessment: From Error Visibility to Structural similarity", IEEE Transactions on Image Processing, Vol. 13, No. 4, April 2004. 